\title{
Spontaneous microstructure formation at water/paraffin oil interfaces
}

\author{
Murielle Schmitt, Ritu Toor, Renaud Denoyel and Mickaël Antoni* \\ Aix-Marseille Univ, CNRS, MADIREL, Marseille, France \\ *m.antoni@univ-amu.fr
}

\begin{abstract}
An experimental investigation of spontaneous emulsification is proposed with water drops pendant in a paraffin oil (PO) solution loaded with a surfactant (SPAN80). Optical microscopy in transmission mode is employed for high spatial resolution image recording. The kinetics of spontaneous emulsification is studied. It is shown to generate a darkening of the drops due to interface modification with a characteristic time that depends upon the SPAN80 concentration. For low concentrations, spontaneous emulsification is slow and produces micrometer sized droplets while for large concentrations, it is fast and bush-like microstructures are observed. These microstructures increase in size and progressively invade the complete water/PO interfaces, detach and finally migrate into the PO phase. This transport phenomenon withdraws water from the drops and leads to a gradual shrinking of their volume. At the end of the process, they appear as deformed objects surrounded by a loose membrane.
\end{abstract}

Keywords: Spontaneous emulsification, water/oil interfaces, SPAN80, microstructures, water transport.

\section{Introduction}

Emulsions are dispersed systems in which non miscible liquids coexist. They can be stabilized by either surface active molecules, solid particles or both surface active molecules and solid particles. Although commonly used in industry for decades, emulsions are still the object of fundamental research activity. Efforts have in particular been devoted to understand the role of solid particles in Ramsden-Pickering emulsions ${ }^{1-3}$, to address stability properties of emulsified dispersions ${ }^{4,5}$ and to the development of low energy processes for nanoemulsion production ${ }^{6,7}$. In this context, intense activity has also been devoted to the description of microemulsions and spontaneous emulsification mechanisms ${ }^{8-12}$. These latter are known to stimulate mass transport phenomena that find applications in a wide range of industrial processes ${ }^{13-15}$.

Conversely to common emulsified systems, microemulsions are thermodynamically stable. As they form spontaneously, no external energy input is necessary. The energy required for emulsification results here only from the reorganization of the chemical compounds at interfaces. The precise underlying dynamic processes are still matter for discussions. To address what happens at liquid/liquid interfaces when spontaneous emulsification (SE) takes place, two main orientations can however be drawn: mechanical breakup and chemical instability. Mechanical breakup can be caused by either interfacial turbulence or vanishing interfacial tensions $^{10,16}$. Chemical instability can be promoted by bilayer collapse due to pressure gradients ${ }^{17,18}$, local inversion of surfactant supersaturated regions ${ }^{19,20}$ or chemical reactivity ${ }^{21}$. When mechanical breakup is the driving phenomenon, low surfactant concentrations are sufficient to evidence SE while if chemical instability prevails, high surfactant concentrations are required. In this last case, it is actually the swelling of inverted micelles due to osmotic pressure that drives $\mathrm{SE}^{19}$. Overall, in spontaneous emulsifying systems, microstructures are formed at the interfaces and their properties can be monitored by the chemicals that are used. They can for example behave as micro-reactors or provide efficient sol-gel synthesis routes for surfactant template-assisted mesostructured materials like for instance solid or hollow spheres ${ }^{22}$, thins films ${ }^{23}$ or high surface area tubules ${ }^{24}$.

The SE phenomena studied in this article generate micro-sized objects that appear, at the macroscopic scale, as cloudy time evolving supernatant phases ${ }^{25}$. SE can therefore be seen as a solubilization process of one 
liquid phase into another. The terminology of microemulsion for surfactant loaded solutions as the one under consideration hereafter is therefore potentially misleading. 'Micellar solutions' might be more adapted since in the conditions of the experiments the PO/SPAN80 solutions are micellar ${ }^{26-28}$. But, as the structures that will be investigated are essentially at the microscale, it is proposed to keep on using the term 'microemulsions' in the following.

SPAN80 is a lipophilic surfactant $(\mathrm{HLB}=4.3)$ consisting mainly is sorbitan monooleate ${ }^{29}$. The properties of water in contact with organic liquids containing SPAN80 were mostly investigated to describe interfacial rheology and emulsion stability ${ }^{5,30-39}$. Important activity has also been devoted to such mixtures in the field of liquid surfactant membranes ${ }^{40}$ that find important applications in extraction for recovery (resp. separation) of valuable (resp. waste) components ${ }^{41-45}$. These latter involve swelling of SPAN80 reverse micelles and mass selective transport mechanisms.

The study of SE kinetics in surfactant-mediated mechanisms is a longstanding problem since water transport into an organic phase has been investigated already more than three decades ago ${ }^{46}$. SE-inducing properties of SPAN80 have been reported for various organic phases ${ }^{47-49}$. Water transfer in water/kerosene/SPAN80 systems and microemulsion formation was recently investigated with optical microscopy in sessile drop configurations ${ }^{50}$. In all these studies, mass transfer rates are shown to be increased by surfactant concentration but, up to now, no discussion is proposed about the influence of the surfactant concentration on the micro-structures in which water is confined. One goal of this paper is to give some insights into this problem.

This work is divided into five sections. After this introduction, the second section will be devoted to material and methods. The third one concentrates on the image processing techniques and presents a contrast function that will be used for the characterization of SE kinetics. The subsequent sections focus on the results and discussions. The formation of SPAN80 driven microstructures is evidenced and their time evolution investigated. It is demonstrated that they progressively withdraw water from the pendant drops. The role of the concentration in SPAN80 and the shape of the microstructures are further discussed. In the last section, conclusions and some perspectives are finally presented.

\section{Materials and Methods}

Experiments are monitored with HPLC grade water and paraffin oil (Fluka 76235) containing varying concentrations of SPAN80 (Sigma-Aldrich 09569) in controlled temperature conditions. Solutions are confined in quartz optical cells (Hellma, 110-QS, $4 \mathrm{~mL}$ ) and analyzed with a microscope in transmission mode. Two different configurations are considered : in configuration A, $2 \mathrm{~mL}$ of PO/SPAN80 solution is gently poured on the top of a $2 \mathrm{~mL}$ water sample (see Figure 1). PO and water are therefore initially in separated phases. In configuration B, a $1.4 \mu \mathrm{L}$ water drop is produced with a Hamilton syringe (Model 62 SYR RN, 2.5 $\mu \mathrm{L}$ ). This latter in mounted on a micrometric support designed to allow accurate positioning. The drop is pendant at the extremity of a needle (see Figure 5) with a hydrophobic tip (22s gauge, Small Hub RN NDL) in the PO/SPAN80 solution. The SPAN80 concentration ranges between 1 and $10 \mathrm{~g} / \mathrm{L}$. Temperature is controlled with a Peltier facility and a water circulating thermostatic bath.

The microscope used is a transmission optical microscope associated with a camera (Mikrotron MC1310). Acquisition frame rate is set to $25 \mathrm{image} / \mathrm{s}$ and each image corresponds to a $1 \times 1 \mathrm{~mm}^{2}$ field of view (FOV) encoded with $1024 \times 1024$ pixels and grey level (GL) values between 0 (black) and 255 (white). Optical calibration ratio is therefore about $1 \mu \mathrm{m}^{2}$. This actually determines the spatial resolution of the microscope, that is to say, the surface of the FOV captured by each pixel. Further 2D calibration factor is not useful here as the 
camera operates square sensors. Depth of field is $30 \mu \mathrm{m}$ and light source is a LED focused at infinity. Such a collimated light is necessary here since all observations are done in transmission mode. These optical constraints allow a good control of vignetting and therefore produce uniformly illuminated reference FOVs.

Scanning electron microscopy (SEM) will also be used in this work. Here, as samples are liquid, the freezefracturing technique is employed. For each experiment, four replica of about $2 \mathrm{~mm}^{3}$ of the cloudy phase (see Figure 1(b)) are produced and deposited on a brass sample holder. This is done by collecting the samples with a micropipette. Once deposited, the four replica are plunged into slushed nitrogen (at $-196^{\circ} \mathrm{C}$ ) and further transferred into a low pressure preparation chamber. This chamber is integrated with a Philips XL30 SFEG STEM scanning electron microscope equipped with an OXFORD cryogenic stage and an OXFORD EDS analysis system. The procedure followed here prevents sample contamination by atmospheric water. The four replica are then fractured using the tip of a scalpel blade. The SEM images are produced in the back-scattered mode and chemical composition obtained with EDX analysis.

\section{Image processing}

Image are recorded in transmission mode and stored in raw format. The pendant drops yield specific optical signals (see Figure 5) that are processed on the basis of contrast differences. To track the evolution of these latter with time, the following contrast function is introduced:

$$
f(t)=\frac{N(t)-N_{\min }}{N(0)-N_{\min }}
$$

where $t$ is time, $N(t)$ (resp. $N(0)$ ) is the number of pixels of the camera detecting a GL > 128 in the considered FOV at time $t$ (resp. initially). These pixels actually detect only transmitted light. $N_{\min }$ is the minimum value reached by $N(t)$ in the image sequence produced by each experiment. With this definition, $f(t)=1$ initially and $f(t)=0$ when the brightness of the FOV is minimal. A decreasing (resp. increasing) trend of $f(t)$ is therefore the signature of an overall increasing (resp. decreasing) opaqueness of the images.

Each measurement consists in a sequence of 5000 images that are treated with the Image-J (IJ) package. A binarization with threshold fixed at GL=128 is first achieved to identify the regions of the FOV where GL>128. $N(t)$ is then computed for all times and both values of $N(0)$ and $N_{\min }$ determined. Fast Fourier transforms (FFT) are further performed with IJ to compute $2 \mathrm{D}$ power spectra with $512 \times 512$ wave numbers. They rely on the 2D Fast Hartley Transform algorithms ${ }^{51,52}$. As power spectra are symmetric, radial averages are finally achieved with a homemade data treatment code to produce radial power spectra.

\section{Qualitative study of water transport}

To show the basic features under study here a first campaign of experiments is presented for configuration A. Temperature is set to $20^{\circ} \mathrm{C}$. Figure 1 illustrates what happens 8 hours after sample preparation for two experiments. The bottom phase of both images is water and the top one is the PO solution. In (a) PO is surfactant free while in (b) it is loaded with SPAN80 (4 g/L) for which interfacial tension is about $3 \mathrm{mN} / \mathrm{m}^{33}$. The curvature of the interface changes from (a) to (b) due to interfacial tension lowering by SPAN80. Initially, the PO phase is transparent for both experiments. Figure 1(a) shows that it remains like this in the absence of 
SPAN80 even after 8 hours. This is clearly not the case when SPAN80 is used. As time runs, a cloudy phase is indeed progressively invading the PO phase as can be observed in Figure 1(b). This turbid phase first appears as a white skin in the vicinity of the water/PO interface. As time runs, it diffuses into the PO phase. Surprisingly, this diffusion takes places not only from the water/PO interface but also from the walls of the optical cell. This can be checked again in Figure 1(b) that shows a rather concave profile of the cloudy phase. Transport of water seems therefore also involving wetting phenomena between water and the quartz cell. A similar effect has been evidenced elsewhere ${ }^{50}$. Its main consequence is to considerably increase the exchange surface where SE can take place and therefore to speed up the overall increasing cloudiness of the samples. Wetting phenomena actually strongly depend on the composition of the solutions and are not easy to control. This is why the pendant drop configuration (configuration B) will be preferred when focusing on the kinetics of water transport.

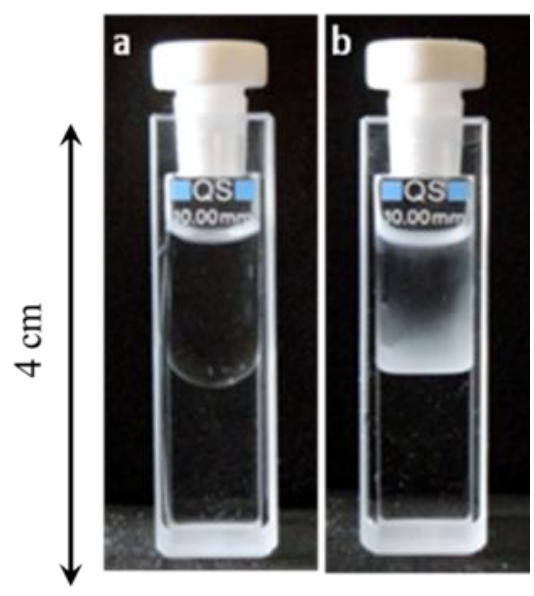

Figure 1: Illustration of water transport into paraffin oil 8 hours after sample preparation. (a) [SPAN80]=0 g/L, (b) [SPAN80] $=4 \mathrm{~g} / \mathrm{L}$. The bottom (resp. top) phase is water (resp. PO). Volume is $2 \mathrm{~mL}$ for both phases.

The above experiments were reproduced for smaller SPAN80 concentrations. Figure 2 shows micrographs of the supernatant phase right above the water/PO interface 24 hours after sample preparation. For [SPAN80] $=5.010^{-3} \mathrm{~g} / \mathrm{L}$ there is no clear evidence of a dispersed phase formation while for larger concentrations $\left(2.510^{-2} \mathrm{~g} / \mathrm{L}\right.$ and $\left.4.2510^{-2} \mathrm{~g} / \mathrm{L}\right)$ a cloudy supernantant phase is present. It contains particles of typical size of few microns. Ref. ${ }^{33}$ indicates that for such concentrations, interfacial tension ranges between $35 \mathrm{mN} / \mathrm{m}$ (for [SPAN80] $=5.010^{-3} \mathrm{~g} / \mathrm{L}$ ) and $15 \mathrm{mN} / \mathrm{m}$ (for [SPAN80]=4.25 $10^{-2} \mathrm{~g} / \mathrm{L}$ ). This should provide sufficient stability of the interface against perturbations and therefore production of microstructures. But, surprisingly, this is clearly not the case here. Their precise shape is not acessible due to the limited resolution of the microscope (1 $\mu \mathrm{m})$. To overcome this problem, cryo-SEM experiments have been achieved.

Figure 3 displays a typical cryo-SEM image of a particle of the supernatant phase of Figure 1(b). It evidences micron-sized droplets that furthermore appear as multiplet-like microstructures (Figure 3(a)). They're composed of water as demonstrated by the intense oxygen peak in the EDX spectrum (Figure 3(b)). This latter is detectable only when focusing the electron beam on microdroplets and vanishes elsewhere. The oxygen atoms of the polar group of the SPAN80 molecules therefore do not generate any measurable EDX response. The SEM analysis demonstrates that SE gives rise to the spontaneous formation of water microdroplets and therefore suggests micellar transport mechanisms. One striking feature here is the complexity of their surface. They appear rough and covered by small polymorphous objects with micrometric sizes that look similar to the ones already evidenced in previous works for high concentrated solutions ${ }^{18,35}$. Local inversion of surfactant supersaturated regions could therefore be one possible driving mechanism here ${ }^{19,20}$. 


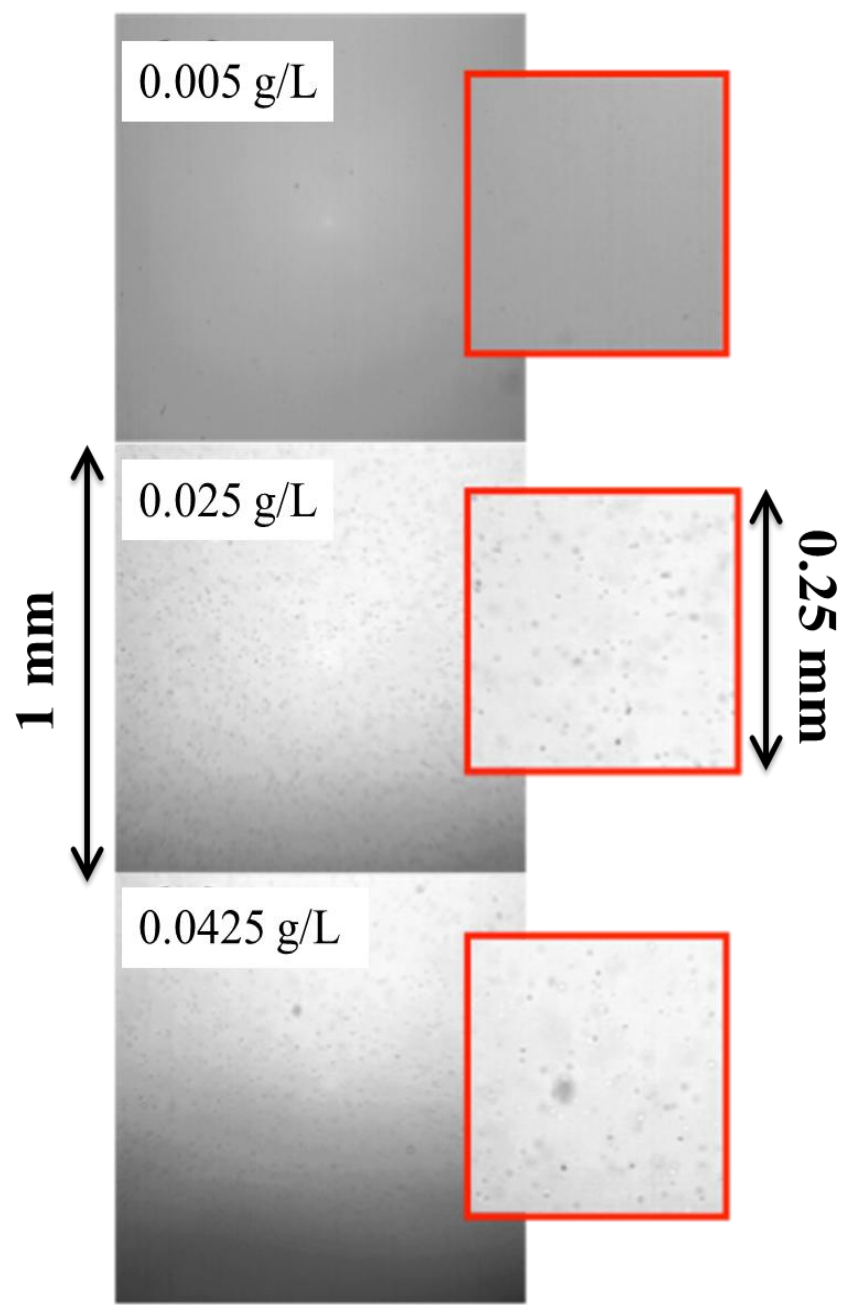

Figure 2: Left: $1 \mathrm{~mm}^{2}$ micrographs of the supernatant phase in the vicinity of the water/PO interface for three values of [SPAN80], 24 hours after sample preparation. SPAN80 concentrations are indicated in the images. Right: magnifications of the left images. Floating objects with micrometric size are visible for [SPAN80] $=0.025 \mathrm{~g} / \mathrm{L}$ and $0.0425 \mathrm{~g} / \mathrm{L}$.

The processes at the origin of the protruding structures of Figure 3(a) and Figure 4 are not clear and should be addressed carefully in particular when operating with freeze fracturing. This technique is indeed known to produce artifacts when not applied in well controlled conditions. Microstructures can for example artificially form due to solidification fronts when dealing with nanoparticle suspensions ${ }^{\mathbf{5 3}}$. Unwanted presence of water due to uncontrolled humidity conditions can moreover give rise to ice crystallites that produce images very similar to the ones of Figure 3(a). Here, experimental conditions were controlled using a transfer chamber where all samples were prepared. The contamination by any external humidity source is hence negligible. The protrusions of Figure 3(a) can therefore be seen as the result of the early stage SE mechanisms. Their number increases with the SPAN80 concentration with a clear hierarchy in their size. Water microdroplets are covered with submicron droplets that are themselves covered with even smaller droplets. This is observable on the top of the largest droplet of Figure 3(a) (red arrow) and in Figure 4. Similar submicron particles are known to be formed in SE either by polymers precipitation, self-assembly of macromolecules or polyelectrolyte complexes $^{\mathbf{1 8 , 3 5 , 5 4}}$. Submicron water droplets seem to be only loosely attached to the interface. Cryo-SEM images indeed show from time to time such objects nearby the largest microdroplets on the PO phase (white arrow in Figure 3). 

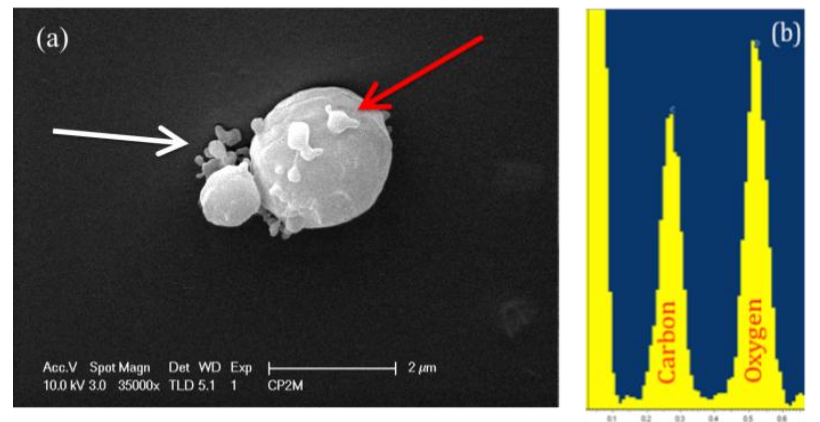

Figure 3 : (a) Cryo-SEM view of a microdroplet of the supernatant phase of Figure 1(b). (b) EDX spectrum. The oxygen peak is a signature of the presence of water. The white (resp. red) arrow in (a) shows submicronic droplets nearby (on the top) of the largest droplet.

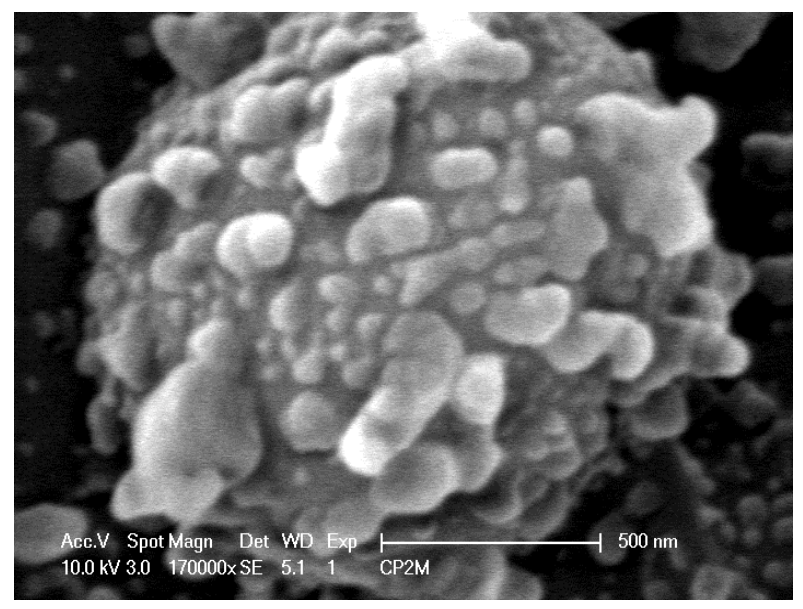

Figure 4 : Detail of the water/PO interface of water microdroplets. Sample is the same than the one used for Figure 3.

\section{Spontaneous emulsification of pendant droplets}

Figure 1(b) demonstrated that wetting phenomena between water and the quartz cell might not be negligible in the experimental conditions. In order to avoid this problem experiments are, from now up, performed using a pendant drop (ie. configuration B). This configuration presents also the important advantage to ease image analysis. For large interfacial tensions, pendant drops have indeed an axial geometry and therefore generate a symmetric optical signal easy to treat when using transmission microscopy. Figure 5(a) shows a typical micrograph right after the injection of the drop in the PO/SPAN80 solution. Its diameter is about $1 \mathrm{~mm}$. In this image, the extremity of the hydrophobic capillary tip is partially visible at the apex of the drop. In the following, temperature is set to $40^{\circ} \mathrm{C}$ for all the experiments.

Figure 5 illustrates the typical time evolution of a drop when [SPAN80]=10 g/L. Relatively large SPAN80 concentrations are used in this section $(1 \mathrm{~g} / \mathrm{L} \leq[\mathrm{SPAN} 80] \leq 10 \mathrm{~g} / \mathrm{L})$ to speed up SE and to ensure a better thermal control for the complete duration of the experiments. For such large concentrations, interfacial tension is reduced down to $3 \mathrm{mN} / \mathrm{m}^{33}$. As time runs, the drop opacity increases but its axi-symmetry is preserved (Figure 5(b)). For long time scales, this symmetry is lost and the drop shows up strongly deformed (see Figure 5(c)). The images of Figure 5 suggest two successive evolution regimes: a first regime denoted $\mathrm{R}_{1}$ where the 
drop darkens but remains axi-symmetric and a second one, $\mathrm{R}_{2}$, where the maximum drop opacity is reached but where axi-symmetry is lost. $\mathrm{R}_{1}$ and $\mathrm{R}_{2}$ are retrieved for all the investigated SPAN80 concentrations-

In $\mathrm{R}_{1}$, the contrast change from Figure 5(a) to Figure 5(b) results from SE that leads to the formation of interfacial microstructures. They consist either water microdroplets (Figure 9) or in microfibers (see Figure 10) that progressively increase in size and invade the complete water/PO interface. As time runs, they form an increasingly dense layer from which they either diffuse into the PO phase or flow down along the interface to the drop bottom where they detach. The overall consequence of this evolution is an increasing opaqueness of the images. In $\mathrm{R}_{1}$, despite $\mathrm{SE}$ and interface crowding, axi-symmetry is preserved and therefore capillary forces prevail. Moreover, as the overall drop size is not significantly modified, interfacial tension remains almost unchanged.
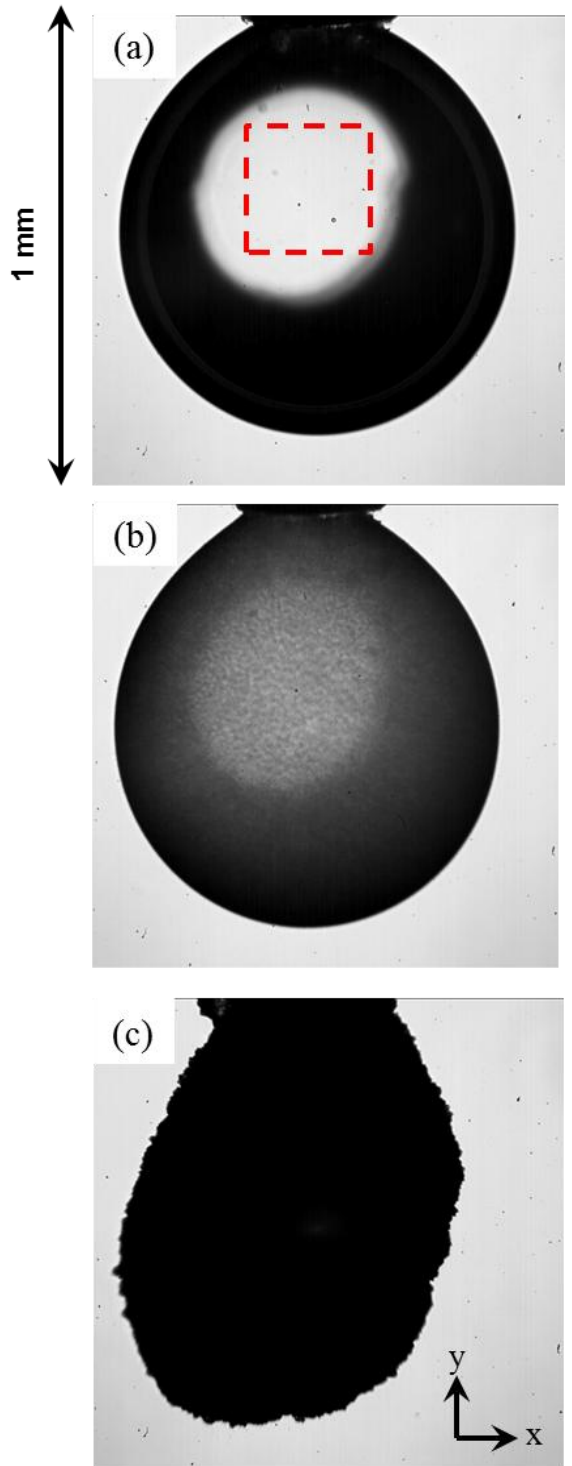

Figure 5 : Shape of the pendant drop for [SPAN80] $=10 \mathrm{~g} / \mathrm{L}$ at different evolution times. (a) $t=0 \mathrm{~s}$, (b) $\mathrm{t}=500 \mathrm{~s}$ and (c) $\mathrm{t}=$ $79400 \mathrm{~s}$. The dashed square in (a) shows the region of interest used for Fourier transform (see Figure 6). Its side is about $0.256 \mathrm{~mm}$.

The typical velocity of the downward capillary flow actually slows down. This suggests the occurrence of larger interfacial viscosities as time runs. At maximal packing, microstructures start to come into contact and hence generate networked and entangled objects. This is what defines the beginning of regime $\mathrm{R}_{2}$. In this regime, 
the capillary flow vanishes but, as the densely packed microstructures are lipophilic (due to large SPAN80 concentration), diffusion into PO continues. Water is therefore continuously withdrawn from the drop and transported into the PO phase leading to a progressive shrinking of its volume. Two coexisting transport mechanisms therefore come into play here: the first one involves water and the diffusion of surfactant molecules that feed SE. This occurs at the molecular scale. The second is at the origin of the capillary flow and therefore involves hydrodynamic scales. This flow drives the microstructures away from the drop interface. On long time scales, water transport is slowed down and finally stops because the interface finally acts like a barrier to any further water removal. The mass transfer rate actually depends on the SPAN80 concentration (see below). When it finally stops, the drop appears as a vesicle with a very rough interface (see Figure 5(c), Figure 9 and Figure 10) that turns out to behave as a loose thin film with weak mechanical resistance.

The patterns distinguishable in the center of Figure 5(b) are actually generated by time evolving microstructure assemblies. They result from both the progressive crowding of the drop interface and flocculation. They are analyzed with a 2D fast Fourier transform in the region of interest (ROI) bounded by the dashed square in Figure 5(a). The resulting power spectrum is plotted in Figure 6(a) where $k_{x}$ and $k_{y}$ are the wave numbers associated to directions $\mathrm{x}$ and $\mathrm{y}$ respectively (see bottom left of Figure 5(c)). Both take value in the interval $[-256,+256] . \mathrm{k}_{\mathrm{x}}= \pm 256$ (resp. $\mathrm{k}_{\mathrm{y}}= \pm 256$ ) corresponds therefore to the wave vector of the full horizontal (resp. vertical) side of the ROI (about $0.256 \mathrm{~mm}$ ). The low contrast of the image in the ROI makes Figure 6(a) rather noisy but some relevant information can still be extracted from it. It shows for instance a shaded circular area in its center (see arrow in Figure 6(a)). This is the signature of objects having a specific size. It becomes more pronounced when performing a radial average. Such an average is reasonable here as the power spectrum is rotation invariant. It is represented in Figure 6(b) for $\mathrm{t}=0 \mathrm{~s}, 500 \mathrm{~s}$ and $1200 \mathrm{~s}$ where $A$ is the amplitude of the Fourier modes. At time $\mathrm{t}=0 \mathrm{~s}$ and $\mathrm{t}=1200 \mathrm{~s}$ curves are similar as the ROI is homogenous (either white or black). For $\mathrm{t}=500 \mathrm{~s}$, larger values of $A$ are evidenced in the range $25<\mathrm{k}<175$ (with $k=\sqrt{k_{x}^{2}+k_{y}^{2}}$ ). Owing to the size of the ROI and to the optical calibration ratio, this wave number domain corresponds to objects of size ranging between $25 \mu \mathrm{m}$ and $175 \mu \mathrm{m}$. This size range is rather broad and suggests that similarly to the hierarchical structures discussed in Figure 3 and Figure 4, larger scale objects, like the flocculated microstructures, do also exhibit complex shapes. Figure 6(b) shows moreover a shoulder at $\mathrm{k} \approx 50$ indicating that their typical size is about $50 \mu \mathrm{m}$ when $\mathrm{t}=500 \mathrm{~s}$. Complementary analysis are clearly necessary here to describe in detail the geometry of these microstructures with, for instance, confocal microscopy or high resolution scanning tomography microscopy ${ }^{4,5}$. 


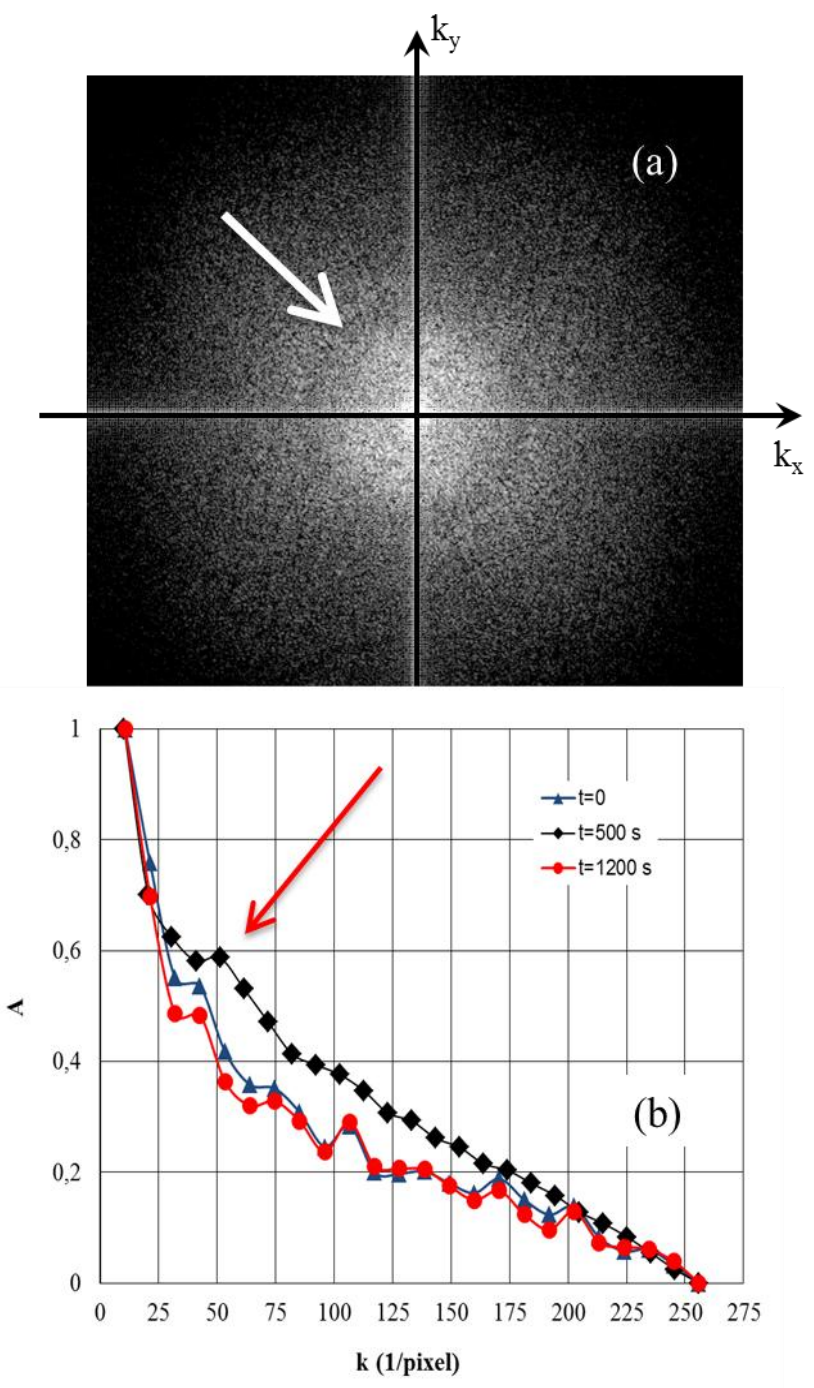

Figure $6:$ (a) power spectrum obtained from the Fourier transform of the square domain in the center of the droplet of Figure 5 (b) at time $\mathrm{t}=500 \mathrm{~s}$. The arrow indicates the circular shaded area. Wave numbers $\mathrm{k}_{\mathrm{x}}$ and $\mathrm{k}_{\mathrm{y}}$ take value in the interval [-256, 256]. Radial average of power spectrum of (a) is plotted in (b) together with the ones obtained at time $t=0 \mathrm{~s}$ and $\mathrm{t}=1200 \mathrm{~s}$. The arrow emphasizes a shoulder at $\mathrm{k} \approx 50$.

Figure 7 is a plot of the contrast function $f(t)$ (see Eq (1)) for four replica experiments realized with [SPAN80] $=10 \mathrm{~g} / \mathrm{L}$. Micrographs are the ones reproduced from Figure 5 and aim to illustrate the link between the drop shape and the corresponding value of $f(t)$. All four replica show similar trends: an early evolution phase where $f(t)$ sharply decays and vanishes at time $t \approx 5000 \mathrm{~s}$. Maximal opaqueness is then attained in the FOV. This first evolution regime actually corresponds to $R_{1}$ discussed previously. In $R_{1}$ experiments show a good reproducibility. For $\mathrm{t}>5000 \mathrm{~s}$, regime $\mathrm{R}_{2}$ starts and $f(t)$ increases back slowly. This is a consequence of the shrinkage of the drop produced by SE and water removal. $\mathrm{R}_{2}$ is emphasized by the grey domain in Figure 7 . This figure also demonstrates that for $\mathrm{t}>15000$ s curves do not overlap anymore. The reason for this is that once SE is fully developed (see Figure 5(c)) microstructures come in contact, the stress along the interface is then strongly modified and becomes anisotropic. This phenomenon is local and appears randomly on the drop interface. It produces drop shapes that change from one experiment to the other since they cannot be controlled with the experimental set up used for this work when long times are considered. Reproducibility is therefore lost. The overall topology of the microstructures is however not affected by this problem. 


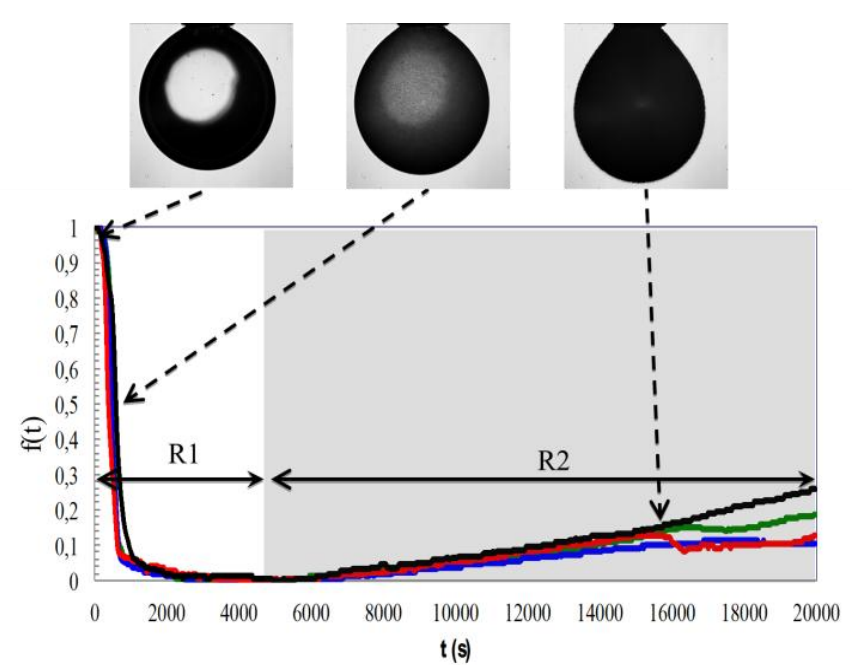

Figure 7 : Evolution of $f(t)$ (see eq. 1) for four replica experiments with [SPAN80] $=10 \mathrm{~g} / \mathrm{L}$. Top images illustrate the typical shape of the drops as time runs. Horizontal arrows indicate the sequences where drops evolve in $R_{1}$ and $R_{2}$ (grey shaded domain).

The way $f(t)$ evolves depends on the SPAN80 concentration as will be shown now. Two characteristic times can be defined here: $t_{1}$ corresponds to the time for which $f\left(t_{1}\right)=1 / 2$ while $t_{2}$ is the time such that $f\left(t_{2}\right)=0$. This latter corresponds to the transition from $\mathrm{R}_{1}$ to $\mathrm{R}_{2} . t_{1}$ and $t_{2}$ describe respectively the short and long time scale processes of SE. Both are plotted in Figure 8. $t_{l}$ monotonously decreases with increasing [SPAN80] and follows with a good accuracy a power law given by :

$$
t_{1}=c_{1}\left(\frac{[\operatorname{SPAN} 80]}{c_{0}}\right)^{-4 / 3}
$$

where $c_{0}$ is a reference concentration $\left(c_{0}=1 \mathrm{~g} / \mathrm{L}\right.$ for units homogeneity) and $c_{1}=1.210^{4} \mathrm{~s}$. The trend for $t_{2}$ is unfortunately not as clear although it also shows a clear decrease for [SPAN80] $<4 \mathrm{~g} / \mathrm{L}$. This is a consequence of the flatness of $f(t)$ when $t \approx t_{2}$ in particular when [SPAN80] $=10 \mathrm{~g} / \mathrm{L}$. 


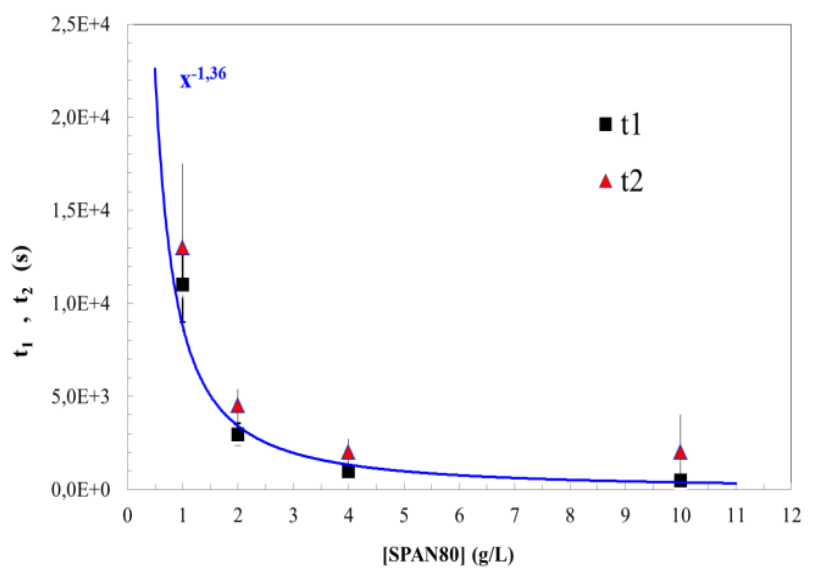

Figure $8: t_{1}$ (squares) and $t_{2}$ (triangles) as a function of [SPAN80]. The full line corresponds to the power law fitting of $t_{1}$ given by Eq. (2). Error bars are computed from the standard error. They're smaller than the size of the markers when not represented.

\section{Interface structure}

This section is devoted to the description of the interface structure for two SPAN80 concentrations. Figure 5 evidenced the progressive modification of the interface that finally turns rough. Figure 6 moreover suggested that SE was generating objects with a typical size of few tens of micrometers. The question is now to get more insights into this roughness and in particular to study how it depends on the SPAN80 concentration. Figure 9 and Figure 10 illustrate the interface structure at time $\mathrm{t}=19000 \mathrm{~s}$ for [SPAN80] $=1 \mathrm{~g} / \mathrm{L}$ and [SPAN80] $=10 \mathrm{~g} / \mathrm{L}$. The drop in Figure 9 is completely opaque. Its interface is covered with a compact layer of almost spherical objects. Although scales are different, they look very similar to the hierarchical microstructures evidenced with cryo-SEM (see Figure 3 and Figure 4). Figure 9(b) shows a zoom of the interface and the arrow a typical example of flocculated microstructures. They're schematically represented in Figure 9(c). Here, SE processes are highly advanced and the interface yet strongly modified. This picture should actually be seen as the result of a dynamic process where the smaller microdroplets pop up on swelling larger ones.

The same evolution is observable for [SPAN80] $=10 \mathrm{~g} / \mathrm{L}$ but instead of microdroplets, the interface is now progressively invaded by fibers as represented in Figure 10. Figure 10(b) shows the detail of the interface and demonstrates that it is now covered by microfibers represented in Figure 10(c). They generate a brushy layer of about $30 \mu \mathrm{m}$ thickness. The microfibers seem to be constituted of assemblies of microdroplets. This assumption is supported by SEM observations that always indicate the formation of microdroplets (see for example Figure 4). The evolution of the microfibers is moreover very similar to the ones of water-dodecanolhexadecane systems where bursting fiber-like objects were also evidenced [18]. When the drop evolves in R1, microfibers grow normally to the interface and therefore make the brushy layer increase in thickness. When their length exceeds $30 \mu \mathrm{m}$, they break and drain downwards into the PO phase. Both effects come in addition to the interfacial top bottom capillary flow discussed above and progressively load the PO phase with a dispersion that becomes clearly visible once $R_{2}$ is reached.

The main outcome when comparing Figure 10 and Figure 9 is the morphology of the microstructures. They turn to strongly depend upon the concentration of SPAN80 and therefore upon its phases in PO. In the concentration range studied here, SPAN80 is most probably organized in complex colloidal structures that yield specific transport patterns. The striking similarity of the microdroplets of Figure 9 and the microstructures illustrated in Figure 3 and Figure 4 suggests that their growing is most probably trigged by an instability that generates, in its early stage, protruding structures on the water/PO interface. 
Figure 9 and Figure 10 actually capture the drop shapes once already deformed and therefore when evolving in regime R2 where reproducibility is lost. But, as already stated above, the microstructures keep their topology from the very beginning of the SE process. So, the microdroplets (resp. microfibers) of Figure 9(b) (resp. Figure 10(b)) are similar to the ones that are already present at time $t_{1}$. The only difference here with earlier times is their packing that turns out to be high enough here to clearly illustrate the interface structure.

For intermediate SPAN80 concentrations ( $2 \mathrm{~g} / \mathrm{L}$ and $4 \mathrm{~g} / \mathrm{L})$, SE leads to the appearance of both microdroplets and microfibers. The transition from interfaces Figure 9 to Figure 10 shows therefore coexisting regimes that could be seen as second order out of equilibrium phase transitions. The specific growing kinetics of each type of microstructures was not precisely investigated so far. Important imaging efforts are indeed necessary to achieve such a goal. One can however reasonably assume here that competing mechanisms are most probably involved to select the topology of the prevailing microstructures while SE is developing. 


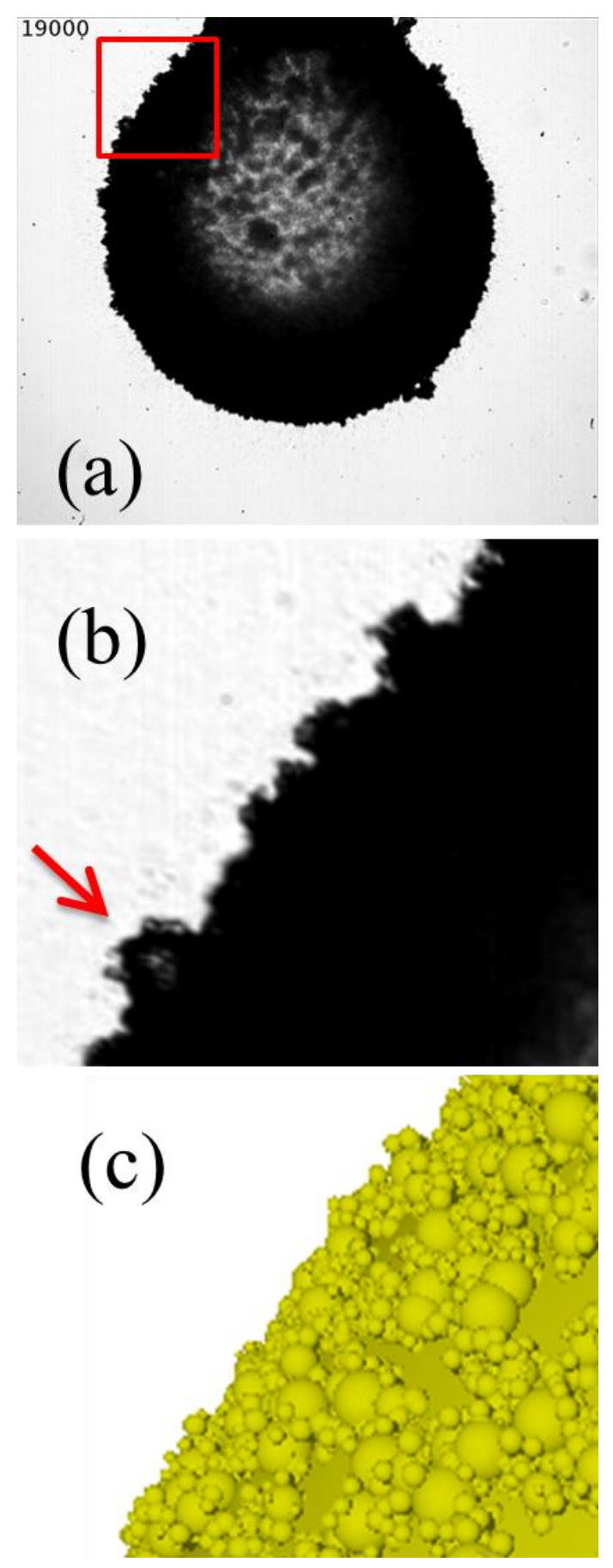

Figure $9:$ (a) shape of the water/PO interface at time $\mathrm{t}=19000 \mathrm{~s}$ for [SPAN80] $=1 \mathrm{~g} / \mathrm{L}$. (b) zoom in the rectangle of (a) showing the presence of microdroplets. The arrow shows a microdroplet of diameter $22 \mu \mathrm{m}$ covered by smaller ones with diameter of about $2 \mu \mathrm{m}$. (c) is a qualitative view of the interface structure.

The precise description of the microscopic mechanisms involved in the SE phenomena of Figure 9 and Figure 10 runs out of the scope of the present work. The phases of SPAN80 when dissolved in PO most probably drastically change topology when concentration is increased from $1 \mathrm{~g} / \mathrm{L}$ to $10 \mathrm{~g} / \mathrm{L}$. With such large concentrations, the PO/SPAN80 solution acts as a surfactant reservoir that drives water transport into PO as long as interface is sufficiently permeable. Transport stops when a membrane-like interface that looks very similar to a jellified phase finally replaces the initial water/PO interface. It acts like a barrier to any further interfacial 
exchanges. The limiting factor of water transport is therefore neither the lack of water in the pendant drop nor insufficient SPAN80 in PO. It seems to be the complete jellification of the water/PO interface that finally interrupts SE and therefore water removal.

To our knowledge, neither the ternary diagram of water/SPAN80/PO mixtures nor microscopic modelling of this system is available in the literature. SPAN80 being itself a non-single component liquid ${ }^{29,37}$ it $^{2}$ would probably make such a description particularly complex since, in principle, its different constituents contribute in different ways in the overall SE. In this context, the different morphologies of the microstructures described above do constitute an interesting signature that could help the understanding of the underlying SE processes. For concentrated solutions, it seems for example reasonable to assume that supersaturated SPAN80 regions in the vicinity of the water/PO interface generate complex reverse micelles that could trigger microstructure formation ${ }^{20}$. These latter could then behave as mobile objects transporting water apart from the pendant drops similarly to what has been observed for capsules ${ }^{21,55,56}$. This scenario can probably be extended to sub-micellar solutions like the ones investigated in Figure 2 where microstructures are also evidenced despite low SPAN80 concentrations. For such solutions, reverse micelles are not stable in the bulk phase since interfacial tension is close to $15 \mathrm{mN} / \mathrm{m}$. This should provide sufficient stability against mechanical perturbations and therefore prevent microstructure formation. The interplay between the different surfactant molecules of SPAN80 seems to still allow local supersaturation regions in particular when in contact with water and therefore promote the occurrence of microstructures. SEM experiments confirm this assessment as they clearly indicate the presence of water microdroplets even for low concentrations (see Figure 3). But one cannot exclude here the formation of SPAN80 flocks that might have sizes below SEM resolution. This last hypothesis needs to be further investigated. 


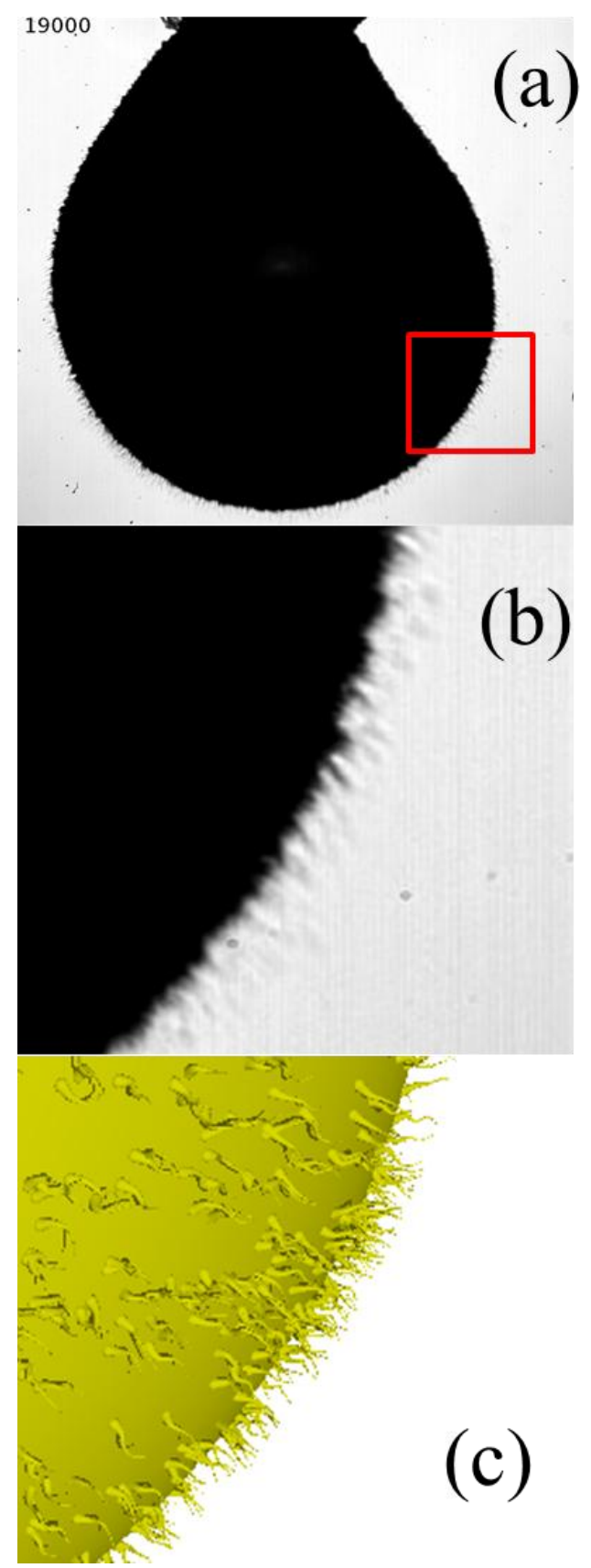

Figure 10 : (a) Shape of the water/PO interface at time $\mathrm{t}=19000 \mathrm{~s}$ for [SPAN80]=10g/L. (b) enlargement of the interface in the rectangle of (a) showing the presence of microfibers. (c) is a qualitative view of the interface structure.

\section{Conclusion and perspectives}

Pendant water drops in a SPAN80 loaded paraffin oil solution are studied. They exhibit spontaneous emulsification processes that drastically modify the structure of water/paraffin oil interfaces even at low SPAN80 concentrations where they should be stable against mechanical perturbations. As time runs, interfaces are spontaneously invaded by growing microstructures that consist in microdroplets and/or microfibers. Image processing and the use of a contrast function allow a qualitative estimation of the characteristic times of this process. They follow a power law decreasing trend when increasing SPAN80 concentration indicating that 
spontaneous emulsification occurs faster at large concentrations. It is further demonstrated that packing of the microstructures increases with time and that they're transported into the paraffin oil phase once spontaneous emulsification is fully developed. This generates a progressive withdrawal of the water from the drops by a mechanism in which water is most probably encapsulated in reverse micelles for SPAN80 concentrated solutions. When this mechanism stops, the drops appear as deformed objects surrounded by a loose membrane. In the system under study in this work, spontaneous emulsification phenomena are most probably the result of the interplay between supersaturated surfactant regions, reserve micelles and vesicle formation. One aim of this work was to propose new experimental insights into this complex problem. It actually demonstrates that spontaneous emulsification can be very efficient for mass transfer across water/paraffin oil interfaces when SPAN80 is used as stabilizer.

\section{Acknowledgments}

Financial support from the European Science Foundation COST Actions MP1106 and CM1101, European Space Agency (projects FASES, PASTA and MATRA), CNES and GdR-CNRS MFA are gratefully acknowledged.

\section{References}

Leal-Calderon, F.; Schmitt, V. Solid-Stabilized Emulsions. Curr. Opin. Colloid Interface Sci. 2008, 13 (4), $217-227 . \quad$ DOI : 10.1016/j.cocis.2007.09.005.

Jiang, J.; Zhu, Y.; Cui, Z.; Binks, B. P. Switchable Pickering Emulsions Stabilized by Silica Nanoparticles Hydrophobized In Situ with a Switchable Surfactant. Angew. Chem. 2013, 125 (47), 12599-12602. DOI : 10.1002/ange.201305947. Emulsion Stabilizers. J. Phys. Chem. B 2015, 119 (20), 6326-6337. DOI : 10.1021/acs.jpcb.5b01805. Dispersed Phase in Water/Oil Emulsions. Langmuir 2014, 30 (16), 4599-4604. DOI : 10.1021/la404766w. Physicochem. Eng. Asp, 2016, 521, 121-132. DOI : 10.1016/j.colsurfa.2016.11.027.

Bouchemal, K.; Briançon, S.; Perrier, E.; Fessi, H. Nano-Emulsion Formulation Using Spontaneous Emulsification: Solvent, Oil and Surfactant Optimisation. Int. J. Pharm. 2004, 280 (1-2), 241-251. DOI : 10.1016/j.ijpharm.2004.05.016.

Sadurní, N.; Solans, C.; Azemar, N.; García-Celma, M. J. Studies on the Formation of O/W Nano-Emulsions, by Low-Energy Emulsification Methods, Suitable for Pharmaceutical Applications. Eur. J. Pharm. Sci. 2005, 26 (5), 438-445. DOI : 10.1016/j.ejps.2005.08.001.

López-Montilla, J. C.; Herrera-Morales, P. E.; Pandey, S.; Shah, D. O. Spontaneous Emulsification: Mechanisms, Physicochemical Aspects, Modeling, and Applications. J. Dispers. Sci. Technol. 2002, 23 (1-3), 219-268. DOI : 10.1080/01932690208984202.

Miller, C. A. Spontaneous Emulsification Produced by Diffusion — A Review. Colloids Surf. A., Physicochem. Eng. Asp, 1988, 29 (1), 89-102. DOI : $10.1016 / 0166-6622(88) 80173-2$. 

275-281. DOI : 10.1006/jcis.1997.4761. 2000; $105,19-72$.

Dabros, T.; Yeung, A.; Masliyah, J.; Czarnecki, J. Emulsification through Area Contraction. J. Colloid Interface Sci. 1999, 210 (1), 222-224. DOI : 10.1006/jcis.1998.5943.

Shahidzadeh, N.; Bonn, D.; Meunier, J. A New Mechanism of Spontaneous Emulsification: Relation to Surfactant Properties. Europhys. Lett. 1997, 40 (4), 459-464. DOI : 10.1209/epl/i1997-00488-0.

(18) Shahidzadeh, N.; Bonn, D.; Meunier, J.; Nabavi, M.; Airiau, M.; Morvan, M. Dynamics of Spontaneous Emulsification for Fabrication of Oil in Water Emulsions. Langmuir 2000, 16 (25), 9703-9708. DOI : 10.1021/la0004931.

(19) Greiner, R. W.; Evans, D. F. Spontaneous Formation of a Water-Continuous Emulsion from a W/O Microemulsion. Langmuir 1990, 6 (12), 17931796. DOI : $10.1021 / \mathrm{la} 00102 \mathrm{a} 014$

(20) Ruschak, K. J.; Miller, C. A. Spontaneous Emulsification in Ternary Systems with Mass Transfer. Ind. Eng. Chem. Fundam. 1972, 11 (4), 534-540. DOI : $10.1021 / 1160044 \mathrm{a} 017$.

(21) Nishimi, T.; Miller, C. A. Spontaneous Emulsification Produced by Chemical Reactions. J. Colloid Interface Sci. 2001, 237 (2), $259-266$. DOI : 10.1006/jcis.2001.7467.

Andersson, N.; Kronberg, B.; Corkery, R.; Alberius, P. Combined Emulsion and Solvent Evaporation (ESE) Synthesis Route to Well-Ordered Mesoporous Materials. Langmuir 2007, 23 (3), 1459-1464. DOI : 10.1021/la0622267.

(23) Walcarius, A.; Sibottier, E.; Etienne, M.; Ghanbaja, J. Electrochemically Assisted Self-Assembly of Mesoporous Silica Thin Films. Nat. Mater. 2007, 6 (8), 602-608. DOI : 10.1038/nmat1951.

(24) Peng, T.; Hasegawa, A.; Qiu, J.; Hirao, K. Fabrication of Titania Tubules with High Surface Area and Well-Developed Mesostructural Walls by Surfactant-Mediated Templating Method. Chem. Mater. 2003, 15 (10), 2011-2016. DOI : 10.1021/cm020828f. Uricanu, V. I.; Duits, M. H. G.; Filip, D.; Nelissen, R. M. F.; Agterof, W. G. M. Surfactant-Mediated Water Transport at Gelatin Gel/Oil Interfaces. J. Colloid Interface Sci. 2006, 298 (2), 920-934. DOI : 10.1016/j.jcis.2006.01.030. Shinoda, K.; Kunieda, H. Conditions to Produce so-Called Microemulsions: Factors to Increase the Mutual Solubility of Oil and Water by Solubilizer. J. Colloid Interface Sci. 1973, 42 (2), 381-387. DOI : 10.1016/0021-9797(73)90303-2. 

Properties of Span-80 Adsorbed Layers at Paraffin-Oil/Water Interface: Capillary Pressure Experiments under Low Gravity Conditions. Colloids Surf. A,. Physicochem. Eng. Asp. 2017. DOI : 10.1016/j.colsurfa.2017.05.012.

(31) Drelich, A.; Gomez, F.; Clausse, D.; Pezron, I. Evolution of Water-in-Oil Emulsions Stabilized with Solid Particles: Influence of Added Emulsifier. Colloids Surf. A, Physicochem. Eng. Asp. 2010, 365 (1-3), 171-177. DOI : 10.1016/j.colsurfa.2010.01.042.

(32) Liu, W.; Sun, D.; Li, C.; Liu, Q.; Xu, J. Formation and Stability of Paraffin Oil-in-Water Nano-Emulsions Prepared by the Emulsion Inversion Point Method. J. Colloid Interface Sci. 2006, 303 (2), 557-563. DOI : 10.1016/j.jcis.2006.07.055.

(33) Santini, E.; Liggieri, L.; Sacca, L.; Clausse, D.; Ravera, F. Interfacial Rheology of Span 80 Adsorbed Layers at Paraffin Oil-water Interface and Correlation with the Corresponding Emulsion Properties. Colloids Surf. A,. Physicochem. Eng. Asp. 2007, 309 (1-3), 270-279. DOI : 10.1016/j.colsurfa.2006.11.041.

(34) Clausse, D.; Drelich, A.; Fouconnier, B. Mass Transfers Within Emulsions Studied by Differential Scanning Calorimetry (DSC) - Application to Composition Ripening and Solid Ripening, Mass Transfer - Advanced Aspects, Dr Hironori Nakajima (Ed.), ISBN: 978-953-307-636-2, 2011. DOI : $10.5772 / 20279$

(35) Koroleva, M. Y.; Yurtov, E. V. Water Mass Transfer in W/O Emulsions. J. Colloid Interface Sci. 2006, 297 (2), 778-784. DOI : 10.1016/j.jcis.2005.10.046

Opawale, F. O.; Burgess, D. J. Influence of Interfacial Properties of Lipophilic Surfactants on Water-in-Oil Emulsion Stability. J. Colloid Interface Sci. 1998, 197 (1), 142-150. DOI : 10.1006/jcis.1997.5222.

Abou-Nemeh, I.; Bart, H. J. Microstructures in the System Water/D2EHPA/Span-80/N-Dodecane. Langmuir 1998, 14 (16), $4451-4459$. DOI : 10.1021/la9710637.

(38) Siladitya, B.; Chatterjee, M.; Ganguli, D. Role of a Surface Active Agent in the Sol-Emulsion-Gel Synthesis of Spherical Alumina Powders. J. SolGel Sci. Technol. 1999, 15 (3), 271-277. DOI : 10.1023/A:1008745210805.

(39) Capdevila, M.; Maestro, A.; Porras, M.; Gutiérrez, J. M. Preparation of Span 80/Oil/Water Highly Concentrated Emulsions: Influence of Composition and Formation Variables and Scale-Up. J. Colloid Interface Sci. 2010, 345 (1), 27-33. DOI : 10.1016/j.jcis.2010.01.045.

(40) Li, N. N. Permeation through Liquid Surfactant Membranes. AIChE J. 1971, 17 (2), 459-463. DOI : 10.1002/aic.690170239.

(41) Chakravarti, A. K.; Chowdhury, S. B.; Chakrabarty, S.; Chakrabarty, T.; Mukherjee, D. C. Liquid Membrane Multiple Emulsion Process of chromium(VI) Separation from Waste Waters. Colloids Surf. A,. Physicochem. Eng. Asp. 1995, 103 (1), 59-71. DOI : 10.1002/aic.690170239. Jiang, C.; Sun, X. Stability of Emulsion Liquid Membrane and Membrane Phase Reaction Spectrum Study of NH3·H2O System. Desalination Water Treat. 2016, 57 (41), 19112-19120. DOI : 10.1080/19443994.2015.1101019.

(43) Mortaheb, H. R.; Amini, M. H.; Sadeghian, F.; Mokhtarani, B.; Daneshyar, H. Study on a New Surfactant for Removal of Phenol from Wastewater by Emulsion Liquid Membrane. J. Hazard. Mater. 2008, 160 (2-3), 582-588. DOI : 10.1016/j.jhazmat.2008.03.095. Xuan-cai, D.; Fu-quan, X. Study of the Swelling Phenomena of Liquid Surfactant Membranes. J. Membr. Sci. 1991, 59 (2), 183-188. DOI : $10.1016 / \mathrm{S} 0376-7388(00) 81182-5$. 25-34. DOI : 10.1016/0376-7388(95)00156-5. DOI : $10.1016 / \mathrm{S} 0376-7388(00) 81330-7$. 
(47) Wen, L.; Papadopoulos, K. D. Visualization of Water Transport in W1/O/W2 Emulsions. Colloids Surf. A,. Physicochem. Eng. Asp. 2000, 174 (1-2), 159-167. DOI : 10.1016/S0927-7757(00)00508-2.

(48) Wen, L.; Papadopoulos, K. D. Effects of Osmotic Pressure on Water Transport in W1/O/W2 Emulsions. J. Colloid Interface Sci. 2001, 235 (2), 398404. DOI : $10.1006 /$ jcis. 2000.7384 .

(49) Villa, L.; Lawson, L. B.; Li, Y.; Papadopoulos, K. D. Internal Coalescence as a Mechanism of Instability in Water-in-Oil-in-Water Double-Emulsion Globules. Langmuir 2003, 19 (2), 244-249. DOI : 10.1021/la026324d.

(50) Silva, P. S.; Zhdanov, S.; Starov, V. M.; Holdich, R. G. Spontaneous Emulsification of Water in Oil at Appreciable Interfacial Tensions. Colloids Surf. A,. Physicochem. Eng. Asp. 2017, 521. DOI : 10.1016/j.colsurfa.2016.05.041.

(51) Bracewell, R. N.; Buneman, O.; Hao, H.; Villasenor, J. Fast Two-Dimensional Hartley Transform. Proc. IEEE 1986, 74 (9), 1282-1283. DOI : 10.1109/PROC.1986.13619.

(52) Hartley, R. V. L. A More Symmetrical Fourier Analysis Applied to Transmission Problems. Proc. IRE 1942, 30 (3), 144-150. DOI : 10.1109/JRPROC.1942.234333.

(53) Limage, S.; Schmitt, M.; Vincent-Bonnieu, S.; Dominici, C.; Antoni, M. Characterization of Solid-Stabilized Water/Oil Emulsions by Scanning Electron Microscopy. Colloids Surf. A,. Physicochem. Eng. Asp. 2010, 365 (1-3), 154-161. DOI : 10.1016/j.colsurfa.2010.02.037. Mora-Huertas, C. E.; Fessi, H.; Elaissari, A. Influence of Process and Formulation Parameters on the Formation of Submicron Particles by Solvent Displacement and Emulsification-diffusion Methods. Adv. Colloid Interface Sci. 2011, 163 (2), 90-122. DOI : 10.1016/j.cis.2011.02.005.

(55) Fessi, H.; Puisieux, F.; Devissaguet, J. P.; Ammoury, N.; Benita, S. Nanocapsule Formation by Interfacial Polymer Deposition Following Solvent Displacement - ScienceDirect. Int. J. Pharm. 1989, 55 (1), R1-R4. DOI : 10.1016/0378-5173(89)90281-0.

(56) Quintanar-Guerrero, D.; Allémann, E.; Doelker, E.; Fessi, H. A Mechanistic Study of the Formation of Polymer Nanoparticles by the EmulsificationDiffusion Technique. Colloid Polym. Sci. 1997, 275 (7), 640-647. DOI : 10.1007/s003960050130. 


\section{For Table of Contents Use Only}
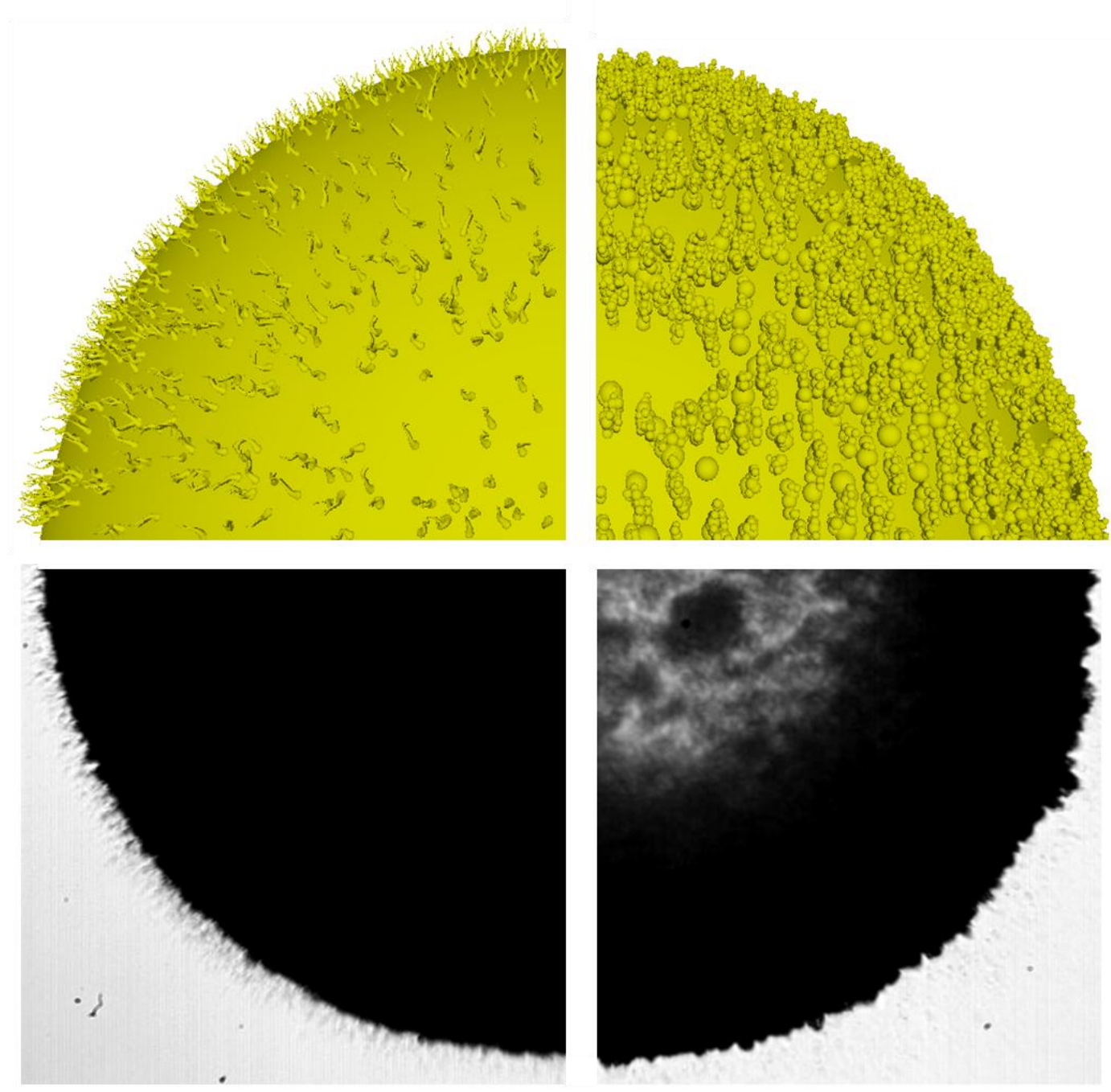